\section{IDDF2019-ABS-0175 DIFFERENCES IN BIOLOGIC BEHAVIORS BETWEEN MIXED AND PURE SIGNET RING CELL CARCINOMA IN CHINESE EARLY GASTRIC CANCER PATIENTS}

Xiao-Yong Wang*, Xiangrong Qin. Department of Gastroenterology, Changzhou No. 2 People's Hospital, Affiliated with Nanjing Medical University, China

\subsection{6/gutjnl-2019-IDDFabstracts. 182}

Background Signet ring cell carcinoma (SRC) of the stomach is a poorly differentiated cancer. Generally, poorly differentiated cancer is believed to show aggressive behavior and poor prognosis. There is no debate about that advanced SRC of the stomach has worse outcomes than does nonsignet ring cell adenocarcinoma (NSRC). However, the behavior of SRC carcinoma in early gastric cancer (EGC) is still controversial. This study aimed to compare clinicopathologic features between SRC and NSRC in Chinese early gastric cancer patients.

Methods We reviewed medical records of patients with EGC who underwent surgical resection between 2009 and 2014 in a tertiary hospital in Jiangsu province, China. We divided EGC into (1) pure SRC carcinoma, which was defined as composed only of signet ring cells (2) mixed SRC carcinoma, defined as signet ring cell carcinoma that was mixed with other histological components and (3) NSRC. We reviewed the clinicopathologic features between these groups.

Results A total of 1008 patients with early gastric cancer were enrolled. 163 patients (16.2\%) were classified as SRC, including 63 cases of pure SRC and 100 mixed SRC. The SRC group was more associated with younger age, female, middle and lower location, mucosa-confined and LNM than NSRC group. Further stratification analyses according to the purity of SRC demonstrated that mixed SRC was associated with an increased risk of LVI $(\mathrm{P}=0.03)$ and $\operatorname{LNM}(\mathrm{P}<0.001)$. In univariate analysis, LNM was associated with mixed SRC histologic type $(\mathrm{P}=0.002)$, submucosal invasion $(\mathrm{P}<0.001)$ and the presence of LVI $(\mathrm{P}<0.001)$. On multivariate logistic regression, the presence of LVI (odds ratio 26.70; 95\% confidence interval 3.01-236.64; $\mathrm{P}=0.003$ ) and submucosal invasion (odds ratio 2.38; 95\% confidence interval 1.01-5.60; $\mathrm{P}$ $=0.04$ ) were independent predictors of LNM in SRC early gastric cancer. No nodal metastases were observed in pure signet ring cell carcinomas $\leq 1 \mathrm{~cm}$ without lymphovascular invasion and confined to the mucosa.

Conclusions Mixed SRC in EGC showed more aggressive behavior than other histologies. Therefore, more attention should be paid to the purity of the SRC carcinoma component in clinical practice.

\section{IDDF2019-ABS-0177 MODULATION OF ANTIMONY MEDIATED THERAPY FOR AN OPTIMAL INSULIN SECRETION DURING VISCERAL LEISHMANIASIS}

Sukrat Sinha*. Nehru Gram Bharati University, India

10.1136/gutjnl-2019-IDDFabstracts. 183

Background Visceral Leishmaniasis is a macrophage-associated disorder for the treatment of which antimony based drug like Sodium Antimony Gluconate has been the first choice in the recent past. About 5 percent of the patients may develop insulin dependent diabetes mellitus. It appears to have a direct action on pancreatic beta cells, resulting in initial insulin release followed by impaired insulin secretion. Within this context, we looked into alternative therapies of treatment along with SAG on triggering the CD2 epitope.

Methods We have evaluated the effect of combining CD2 with conventional antimonial (sb) therapy in protection in $\mathrm{BALB} / \mathrm{c}$ mice infected with either drug sensitive or resistant strain of Leishmania donovani with 3 million parasites via-intra-cardiac route. Mice were treated with anti CD2 adjunct SAG subcutaneously twice a week for $\underline{4}$ weeks. Assessment for measurement of weight, spleen size, anti-Leishmania antibody titer, $\mathrm{T}$ cell and anti-leishmanial macrophage function was carried out day $0,10,22$ and 34 post treatments. Insulin levels were also determined on the same intervals.

Results The combination therapy was shown boosting significant proportion of $\mathrm{T}$ cells to express CD25 compared to SAG monotherapy. Although, the level of IFN- $\gamma$ was not statistically different between combination vs monotherapy $(\mathrm{p}=0.298)$ but CD2 treatment even alone significantly influenced IFN- $\gamma$ production than either SAG treatment $(\mathrm{p}=0.045)$ or with $\mathrm{CD} 2$ adjunct SAG treatment $(\mathrm{p}=0.005)$ in $\mathrm{Ld}-\mathrm{S}$ strain as well as in Ld-R strain. The influence of CD2 adjunct treatment was also documented in anti-leishmanial functions in macrophages. Interestingly insulin levels were observed to be optimal on supplementing SAG along with CD2.

Conclusions SAG along with CD2 could be used as a potential therapy to overcome incidences of Diabetes mellitus during Visceral Leishmaniasis

\section{IDDF2019-ABS-0178 THE PARTICIPATION RATE BETWEEN CT COLONOGRAPHY AND COLONOSCOPY IN SCREENING POPULATION}

${ }^{1} \mathrm{He} \mathrm{Zhu}^{*},{ }^{1}$ Fudong Li, ${ }^{1} \mathrm{Ke}$ Tao, ${ }^{11}$ Jing Wang, ${ }^{2}$ Carissa Scurlock, ${ }^{3}$ Xiaofei Zhang, ${ }^{1}$ Hong Xu. ${ }^{1}$ Department of Gastroenterology, The First Hospital of Jilin University, Changchun, China; ${ }^{2}$ Institute for Social Research, University of Michigan, Ann Arbor, MI, USA; ${ }^{3}$ Department of Clinical Epidemiology and Biostatistics, Beijing Tsinghua Changgung Hospital Affiliated With Tsinghua University, Beijing, China

\subsection{6/gutjnl-2019-IDDFabstracts. 184}

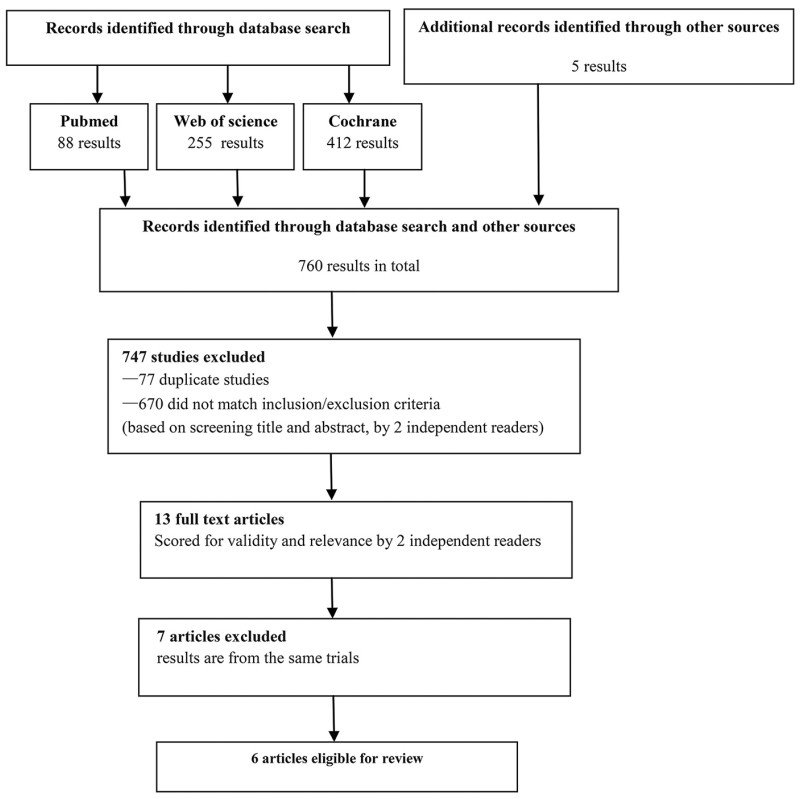

Abstract IDDF2019-ABS-0178 Figure 1 